간호대학생의 임상실습교육에 대한 자기효능감, 전문직 자아개념 및 임상실습만족도

김남희 $1 \neq$, 김경원 ${ }^{2}$

1춘해보건대학 간호과, ${ }^{2}$ 대구한의대학교 간호학과

\title{
Self-Efficacy, Self-Concept of Professional Nursing, and Clinical Practice Satisfaction to Clinical Practice Education in Nursing Students
}

\author{
Nam-Hee Kim ${ }^{1}$, Kyung-Won Kim² \\ ${ }^{1}$ Department of Nursing, Choonhae College of Health Science \\ ${ }^{2}$ Department of Nursing, Dague Hanny University
}

\begin{abstract}
$<$ Abstract $>$
The purpose of this study was to identify self-efficacy and self-concept of professional nursing which might influence clinical practice satisfaction in nursing students. The participants were 278 nursing students in U city. Date were collected from November 29 to December 17, 2010.

The collected data was analyzed with the PASW 18.0 statistics program. Self-efficacy had significant positive correlation with self-concept of professional nursing(r=.595, $\mathrm{p}<.001)$ and self-concept of professional nursing had significant positive correlation with clinical practice satisfaction $(r=.396, p<.001)$. In multiple regression analysis, self-concept of professional nursing is most influential factor explained $21 \%$ of variance of clinical practice satisfaction.

The results indicate that to develop a more reasonable and effective curriculum and teaching program for enhancement of students' self-concept of professional nursing.
\end{abstract}

Key Words : Self-efficacy, Self-concept, Clinical Practice Satisfaction, Nursing Students 


\section{I . 서론}

1. 연구의 필요성

임상실습은 간호학생이 강의에서 배운 지식과 기술을 직접 간호현장에 적용하여 실무능력을 키 우는 실제적인 교육과정이다[1]. 이 과정에서 간호 학생은 실습병동 간호사의 역할을 학습하고 따라 하면서 한사람의 간호사로 성장할 뿐 아니라 대상 자가 가진 건강문제를 찾아내어 중재함으로써 비 판적 사고와 분석적 기술을 익히게 된다. 그리고 대상자와 효과적인 의사소통을 하고 정서적 지지 를 제공하면서 전인적인 간호를 펼칠 수 있는 역 량을 갖추게 된다[2]. 따라서 임상실습은 간호학생 이 졸업 후 바로 임상간호사의 역할을 차질 없이 수행하여 전문직 간호사로서 발전해 나가는데 가 장 핵심 요소라고 할 수 있다.

그러나 임상실습이 이렇게 중요함에도 불구하고 간호학생들의 임상실습에 대한 만족도는 매우 낮 은 것으로 밝혀졌으며, 현재 행해지고 있는 임상실 습은 이론과 실무의 차이, 간호기술을 습득할 수 있는 기회 부족, 임상실습 지도교수 부족, 학생들 의 실습의욕 저하 등 여러 가지 이유로 간호학생 이 전문직 간호사로서 갖추어야 할 지식, 기술 및 태도를 배양하는 데 문제가 되고 있다[3]. 또한 신 설 간호학과 증가, 기존 간호학과의 정원의 증가에 따라 실습학생 수가 증가와 임상실습 현장에서 대 상자들이 학생들이 행하는 간호행위 거부로 인하 여 학생들의 임상실습 경험이 제한되고 있다[4].

간호학생 때의 관찰에 국한된 실습이나 이론 교 육과 실제 임상과의 차이로 인해 간호사들이 희망 과 의욕을 가지고 열심히 하겠다는 의지로 임상에 나가지만 졸업 직후 독립적으로 일할 준비가 되지 않아 임상간호사로서 적응하는데 어려움을 겪게 되며[5], 학생들이 실습교육을 제대로 이행하지 않 은 상황에서 간호사가 되어 바로 환자를 돌보아야
되므로 환자의 안전에 위험요인이 될 수도 있으며 나아가 전문직 간호사의 위상까지 떨어뜨릴 수도 있다. 또 다른 문제는 여러 학교가 한 실습지에 몰 리는 현상이 두드러지면서 실습이 필요한 병동으 로 학생을 보낼 수 없으며 4 년제 간호학과와 3 년 제 간호과가 함께 실습하는 경우에는 학생의 실습 만족도까지 차이가 나고 있다[6]. 그리고 이와 같 은 결과는 향후 간호학과를 졸업하고 임상현장에 나가서 전문간호를 수행해야 하는 간호사를 위축 되게 만들 수 있다.

자기효능감은 어떤 목표를 성취하기 위한 행위 를 수행하는 자신의 능력에 대한 신념으로써 자기 효능감이 낮은 사람은 성공적인 업무 수행에 문제 를 일으킨다[7]. 간호학생들이 임상실습을 보다 만 족스럽고 효율적으로 수행하기 위해서는 임상실습 을 성공적으로 수행할 수 있다는 기대를 가져야 한다[4].

여러 분야의 보건의료 전문인들과 함께 일하는 임상현장에서 전문직 간호사로서 업무를 효율적으 로 수행하기 위해서는 긍정적인 전문직 자아개념 을 확립하는 것이 필요하다[8]. 간호학생은 임상실 습 동안 자신이 생각한 이상적인 간호와 실제로 수행되는 간호에 차이가 있다는 것은 인식하게 되 고 간호에 대한 부정적인 인식이 생기기도 한다고 보고하여 임상실습이 간호전문직 자아개념 발달과 관계가 있다는 것을 언급하였다[9]. 이러한 전문직 자아개념은 간호사의 자존감, 자신감 및 자신에 대 한 자아개념에 영향을 미치고 이는 간호의 생산성 과도 관련이 있는 것으로 나타났다[10].

따라서 임상실습에서 간호학생의 역할수행에 따 른 자기효능감과 전문직 간호사에 대한 인식정도 를 사정해 볼 필요가 있다고 사료된다.

임상실습과 관련하여 기존의 연구에서는 대상자 를 4 년제와 3 년제로 나누어 전문직 자아개념과 실 습만족도, 임상수행능력과 자기효능감을 확인함으 로써[11][12][13] 임상실습의 문제점을 제기하여 왔 
으나 간호학생의 자기효능감과 전문직 자아개념 및 임상실습 만족도를 함께 본 연구는 없었다. 더 욱이 학생 수가 증원되어 실습환경이 더욱 열악해 진 상황에서는 간호사의 양적인 증가를 가져올 수 있으나 간호사의 질적인 향상까지는 보장할 수 없 기 때문에 임상실습을 통해 전문직 간호사의 위상 을 재확립할 수 있는 연구가 더욱 절실하다고 할 수 있다.

이에 본 연구자는 간호학생들의 자기효능감, 전 문직 자아개념 및 임상실습 만족도와의 관계를 파 악하고 자기효능감과 전문직 자아개념이 임상실습 만족도에 미치는 영향정도를 파악하여 간호학생이 졸업 후 임상현장에서 전문직 간호사로서 간호역 량을 충분히 발휘할 수 있도록 하는 실습중재방안 을 모색하고자 한다.

\section{2. 연구의 목적}

본 연구는 간호대학생의 자기효능감, 전문직 자 아개념 및 임상실습 만족도를 파악하고 자기효능 감과 전문직 자아개념이 임상실습 만족도에 미치 는 영향정도를 파악하기 위한 것으로 구체적인 목 적은 다음과 같다.

첫째, 대상자의 자기효능감, 전문직 자아개념 및 임상실습 만족도를 파악한다.

둘째, 대상자의 일반적 특성에 따른 자기효능감, 전문직 자아개념 및 임상실습 만족도의 차이를 파 악한다.

셋째, 대상자의 자기효능감, 전문직 자아개넘 및 임상실습 만족도와의 상관관계를 파악한다.

넷째, 대상자의 자기효능감과 전문직 자아개념 이 임상실습 만족도에 미치는 영향정도를 파악한 다.

\section{ㅍ. 연구방법}

1. 연구설계

본 연구는 간호대학생을 대상으로 자기효능감, 전문직 자아개념 및 임상실습 만족도와의 관계를 파악하고 자기효능감과 전문직 자아개념이 임상실 습 만족도에 미치는 영향정도를 파악하기 위한 서 술적 조사연구이다.

\section{2. 연구대상 및 자료수집 방법}

본 연구의 대상자는 일 광역시에 소재한 1 개 3 년제 간호과에 재학 중인 2학년 3개반 192명, 3학 년 3개반 98 명 총 290 명을 대상으로 하였다. 3학년 은 방학 특강 기간 중에 자료수집을 하여 결석한 학생들이 많아 2학년에 비해 수가 적었다.

자료수집 기간은 2010년 11월 29일부터 12월 17 일까지였다. 연구자가 직접 학생들에게 연구의 목 적과 취지, 연구의 익명성에 대해 설명하고 자발적 으로 참여하기를 원하는 대상자에게 서면 동의서 를 받은 후 자기기입식 설문지를 배부하여 그 자 리에서 회수하였다. 회수된 질문지 중 응답이 불충 분한 자료 12 부를 제외한 278 부를 최종 자료분석 에 사용하였다.

\section{3. 연구도구}

1) 자기효능감

자기효능감은 Sherer 등[14]이 개발하고 홍혜영 [15]이 번안하고 수정한 자기효능감 도구 중 일반 적 자기효능감 17 문항으로 구성된 도구를 사용하 였다. 각 문항은 5 점 척도로 '전혀 그렇지 않다' 1 점에서 '매우 그렇다' 5점으로 점수가 높을수록 자 기효능감이 높음을 의미한다. 홍혜영[15]의 연구에 
서 Cronbach' a는 .86이었고 김영희[13]의 연구에 서 Cronbach' a는 .92였으며 본 연구에서 Cronbach' a는 .90 이었다.

2) 전문직 자아개념

간호전문직 자아개념은 Arthur[10]가 개발한 Professional Self-concept of Nurses Instrument (PSCNI)를 서원희[8]가 간호대학생에게 적합하도록 수정한 도구를 사용하였다. 지도력 5 문항, 간호기 술 4 문항, 융통성 6 문항, 만족감 8 문항, 의사소통 4 문항의 총 27 문항으로 $9,12,13,18,21,23,25$ 번 문항은 역문항으로 처리하였으며 각 문항은 4점 Likert 척도로 '그렇지 않다' 1점에서 '매우 그렇다' 4점으로 점수가 높을수록 전문직 자아개념의 정도 가 높음을 의미한다. 서원희[8]의 연구에서 Cronbach' $\alpha$ 는 .87이었고 본 연구에서 Cronbach' $a$ 는 .75이었으며 하부영역의 Cronbach' $a$ 는 리더 십 .72 , 간호기술 .73 , 융통성 .81 , 의사소통 .50 , 만 족감 .61이었다.

3) 임상실습 만족도

서원희[8]가 사용한 임상실습 만족도 도구로 실 습교과에 대한 만족도 3 문항, 실습내용에 대한 만 족도 6문항, 실습지도에 대한 만족도 9문항, 실습 환경에 대한 만족도 7 문항, 실습시간에 대한 만족 도 3 문항, 실습평가에 대한 만족도 3 문항의 총 31 문항이다. 각 문항은 5점 Likert척도로 '전혀 그렇 지 않다' 1점에서 '매우 그렇다' 5점으로 점수가 높 을수록 임상실습 만족도가 높음을 의미한다. 서원 희[8]의 연구에서 Cronbach' a는 .89이었고, 본 연 구에서 Cronbach' a는 .92이었으며 하부영역의 Cronbach' a는 실습교과 .66, 실습내용 .55 , 실습지 도 .86 , 실습환경 .76 , 실습시간 .88 , 실습평가 .70 이 었다.
4. 자료분석 방법

수집된 자료는 PASW 18.0 프로그램을 이용하 여 분석하였으며 구체적인 방법은 다음과 같다. 대 상자의 일반적 특성은 실수와 백분율, 평균과 표준 편차를 구하였고, 자기효능감, 전문직 자아개념 및 임상실습 만족도는 평균과 표준편차를 구하였다. 또한 대상자의 일반적 특성에 따른 자기효능감, 전 문직 자아개념 및 임상실습 만족도의 비교는 t-test, ANOVA를 시행하였고, 변수들 간의 상관관 계는 Pearson's correlation을 실시하였다. 마지막으 로 자기효능감과 전문직 자아개념이 임상실습만족 도에 주는 영향 정도를 파악하기 위해 Multiple regression을 실시하였다.

5. 연구의 제한점

본 연구는 일개 전문대학 간호과 학생을 대상으 로 하였으므로 연구의 일반화에 제한점이 있다.

\section{III. 연구결과}

1. 대상자의 일반적 특성

본 연구의 대상자는 총 278 명으로 여학생이 $90.6 \%$ 이었고, 남학생은 $9.4 \%$ 이었다. 학년은 2 학년 이 $69.1 \%$ 이고 3 학년이 $30.9 \%$ 이었으며, 대상자의 연령은 19세부터 38세까지 분포하였고, 평균연령은 22.06 세였다. 대상자의 종교는 무교가 $46.8 \%$ 로 가 장 많았고, 건강상태는 $79.5 \%$ 가 좋다고 답하였며, 대학생활은 $53.3 \%$ 가 만족해하였고, 학교성적은 중 간이 $70.5 \%$ 로 가장 많았다. 간호과 지원동기는 취 업률이 높아서가 $46.0 \%$ 로 가장 많았고, 적성과 취 미에 맞아서 $23.0 \%$, 부모님의 권유에 의해서 
$19.8 \%$ 의 순이었다. 간호학 전공에서는 $69 \%$ 가 만족 하였고, 실습량에서 $45 \%$ 그리고 간호학생에 대한
환자의 인식과 태도에서는 $53.2 \%$ 가 만족한다고 답 하였다<표 1>.

<표 1> 대상자의 일반적 특성

단위: 명 $(\%)$

\begin{tabular}{|c|c|c|c|}
\hline 특성 & 구분 & 명 & $\%$ \\
\hline \multirow{2}{*}{ 성별 } & 여자 & 252 & 90.6 \\
\hline & 남자 & 26 & 9.4 \\
\hline \multirow{2}{*}{ 학년 } & 2학년 & 192 & 69.1 \\
\hline & 3학년 & 86 & 30.9 \\
\hline \multirow{3}{*}{ 연령 } & 25세 미만 & 228 & 82.0 \\
\hline & 25세이상-29세 이하 & 34 & 12.2 \\
\hline & 30세 이상 & 16 & 5.8 \\
\hline \multirow{5}{*}{ 종교 } & 불교 & 65 & 23.4 \\
\hline & 기독교 & 56 & 20.1 \\
\hline & 카톨릭 & 15 & 5.4 \\
\hline & 없음 & 135 & 48.6 \\
\hline & 기타 & 7 & 2.5 \\
\hline \multirow{3}{*}{ 건강상태 } & 좋다 & 221 & 79.5 \\
\hline & 보통이다 & 43 & 15.5 \\
\hline & 나쁘다 & 14 & 5.0 \\
\hline \multirow{3}{*}{ 대학생활 만족도 } & 만족하다 & 154 & 53.3 \\
\hline & 보통이다 & 103 & 37.0 \\
\hline & 불만족하다 & 27 & 9.7 \\
\hline \multirow{3}{*}{ 학교성적 } & 상 & 42 & 15.1 \\
\hline & 중 & 196 & 70.5 \\
\hline & 하 & 40 & 14.4 \\
\hline \multirow{7}{*}{ 간호과 지원동기 } & 고교성적을 고려해서 & 6 & 2.2 \\
\hline & 적성과 취미에 맞아서 & 64 & 23.0 \\
\hline & 취업률이 높아서 & 128 & 46.0 \\
\hline & 부모님의 권유로 & 55 & 19.8 \\
\hline & 봉사직을 갖기 위해 & 8 & 2.9 \\
\hline & 해외로 갈 기회가 많아서 & 4 & 1.4 \\
\hline & $\begin{array}{l}\text { 본인이 가족의 질환이나 입원시에 받은 인상 때 } \\
\text { 문에 }\end{array}$ & 13 & 4.7 \\
\hline \multirow{3}{*}{ 간호학 전공에 대한 만족도 } & 만촉하다 & 192 & 69.0 \\
\hline & 보통이다 & 77 & 27.7 \\
\hline & 불만족하다 & 9 & 3.3 \\
\hline \multirow{3}{*}{ 실습량에 대한 만족도 } & 만족하다 & 125 & 45.0 \\
\hline & 보통이다 & 105 & 37.8 \\
\hline & 불만족하다 & 48 & 17.2 \\
\hline \multirow{3}{*}{$\begin{array}{l}\text { 간호학생에 대한 환자의 } \\
\text { 인식과 태도 }\end{array}$} & 만족하다 & 148 & 53.2 \\
\hline & 보통이다 & 99 & 35.6 \\
\hline & 불만족하다 & 31 & 11.2 \\
\hline
\end{tabular}


2. 대상자의 자기효능감, 전문직 자아개념 및 임상실습 만족도

연구대상자의 자기효능감 평균은 $3.50 \pm .59$ 점이 었고 전문직 자아개념의 평균은 $2.85 \pm .34$ 이었으며 전문직 자아개념의 가장 높은 영역은 간호기술로 $2.94 \pm .46$ 점, 가장 낮은 영역은 리더십으로 $2.79 \pm .42$ 점으로 나타났다. 임상실습 만족도의 평균은 $3.10 \pm .56$ 점이었고 가장 높은 영역은 실습교과로 $3.54 \pm .75$ 점, 가장 낮은 영역은 실습지도로 $2.88 \pm .79$ 점으로 나타났다<표 2>.

<표 2> 대상자의 자기효능감, 전문직 자아개념 및 임상실습 만족도의 정도

\begin{tabular}{clc}
\hline 특성 & 항목 & 평균 및 표준편차 \\
\hline \hline 자기효능감 & & $3.50 \pm .59$ \\
\hline & 리더십 & $2.79 \pm .42$ \\
& 간호기술 & $2.94 \pm .46$ \\
전문진 & 융통성 & $2.91 \pm .43$ \\
자애념 & 의사소통 & $2.84 \pm .45$ \\
& 만족감 & $2.80 \pm .45$ \\
& 소계 & $2.85 \pm .34$ \\
\hline & 실습교과 & $3.54 \pm .75$ \\
& 실습내용 & $3.05 \pm .55$ \\
& 실습지도 & $2.88 \pm .79$ \\
임상실습 & 실습환경 & $3.25 \pm .61$ \\
만족도 & 실습시간 & $3.06 \pm .88$ \\
& 실습평가 & $3.05 \pm .81$ \\
& 소계 & $3.10 \pm .56$ \\
\hline
\end{tabular}

3. 대상자의 일반적 특성에 따른 자기효능감, 전문직 자아개념 및 임상실습 만족도

대상자의 자기효능감은 연구대상자의 성별 $(\mathrm{t}=3.151, \mathrm{p}=.002)$, 학년 $(\mathrm{t}=-3.201, \mathrm{p}=.002)$, 건강상태
$(\mathrm{F}=7.433, \mathrm{p}=.001)$, 대학생활의 만족도( $\mathrm{F}=10.465$, $\mathrm{p}<.001)$, 학교성적( $\mathrm{F}=9.829, \mathrm{p}<.001)$, 간호학 선택동 기 $(\mathrm{F}=2.389, \mathrm{p}=.029)$, 간호학 전공에 대한 만족도 $(\mathrm{F}=18.776, \mathrm{p}<.001)$, 및 실습량에 대한 만족도 $(\mathrm{F}=7.546, \mathrm{p}=.001)$ 와 통계적으로 유의한 차이가 있 었다. 그러나 대상자의 연령 $(\mathrm{F}=2.541, \mathrm{p}=.081)$, 종교 $(\mathrm{F}=.382, \mathrm{p}=.821)$, 그리고 간호학생에 대한 환자의 인식과 태도( $\mathrm{F}=.171, \mathrm{p}=.843)$ 에 따른 자기효능감은 차이가 없는 것으로 나타났다.

대상자의 전문직 자아개념은 성별 $(\mathrm{t}=2.912$, $\mathrm{p}=.004)$, 학년 $(\mathrm{t}=-3.869, \mathrm{p}<.001)$, 건강상태 $(\mathrm{F}=7.497$, $\mathrm{p}=.001)$, 대학생활의 만족도( $\mathrm{F}=16.580, \mathrm{p}<.001)$, 학 교성적 $(\mathrm{F}=3.107, \mathrm{p}=.046)$, 간호학 선택동기 $(\mathrm{F}=5.076$, $\mathrm{p}<.001)$, 간호학 전공에 대한 만족도 $(\mathrm{F}=32.331$, $\mathrm{p}<.001)$, 실습량에 대한 만족도( $\mathrm{F}=14.763, \mathrm{p}<.001)$, 간호학생에 대한 환자의 인식과 태도 $(\mathrm{F}=4.319$, $\mathrm{p}=.014)$ 에서 통계적으로 유의한 차이가 있었다. 대 상자의 연령 $(\mathrm{F}=2.136, \mathrm{p}=120)$ 과 종교 $(\mathrm{F}=1.252$, $\mathrm{p}=.289$ )는 전문직 자아개념과는 통계적으로 유의하 지 않았다.

대상자의 임상실습 만족도는 성별 $(\mathrm{t}=3.022$, $\mathrm{p}=.003)$, 간호학 전공에 대한 만족도 $(\mathrm{F}=6.112$, $\mathrm{p}=.003)$, 실습량에 대한 만족도( $\mathrm{F}=12.390, \mathrm{p}<.001)$, 간호학생에 대한 환자의 인식과 태도 $(\mathrm{F}=5.790$, $\mathrm{p}=.003$ )에서 통계적으로 유의한 차이가 있었다. 그 러나 대상자의 연령 $(\mathrm{F}=.210, \mathrm{p}=.811)$, 학년 $(\mathrm{t}=-1.580$, $\mathrm{p}=115)$, 종교 $(\mathrm{F}=1.413, \mathrm{p}=.230)$, 건강상태 $(\mathrm{F}=1.343$, $\mathrm{p}=.263)$, 대학생활의 만족도 $(\mathrm{F}=2.868, \mathrm{p}=.059)$, 학교 성적 $(\mathrm{F}=.423, \mathrm{p}=.656)$, 간호학 선택동기 $(\mathrm{F}=1.333$, $\mathrm{p}=.243$ )에서는 유의하게 차이가 없었다<표 3>. 
<표 3> 대상자의 일반적 특성에 따른 자기효능감, 전문직 자아개념 및 임상실습 만족도의 차이

\begin{tabular}{|c|c|c|c|c|c|c|c|}
\hline \multirow[b]{2}{*}{ 특성 } & \multirow{2}{*}{ 범주 } & \multicolumn{2}{|c|}{ 자기효능감 } & \multicolumn{2}{|c|}{ 전문직 자아개념 } & \multicolumn{2}{|c|}{ 임상실습 만족도 } \\
\hline & & $\begin{array}{l}\text { 평균 및 } \\
\text { 표춘편차 }\end{array}$ & $\begin{array}{c}t \text { or } F \\
(p)\end{array}$ & $\begin{array}{l}\text { 펵균 및 } \\
\text { 표춘편차 }\end{array}$ & $\begin{array}{c}t \text { or } F \\
(p)\end{array}$ & $\begin{array}{l}\text { 펵균 및 } \\
\text { 표춘편차 }\end{array}$ & $\begin{array}{l}t \text { or } F \\
(p)\end{array}$ \\
\hline \multirow[t]{2}{*}{ 성별 } & 여자 & 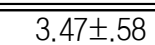 & 3.151 & $2.83 \pm .34$ & 2.912 & 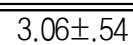 & 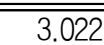 \\
\hline & 남자 & $3.85 \pm .62$ & $(.002)$ & $3.03 \pm .33$ & $(.004)$ & $3.40 \pm .67$ & $(.003)$ \\
\hline \multirow[t]{2}{*}{ 학년 } & 2학년 & $3.43 \pm .60$ & -3.201 & $2.80 \pm .33$ & -3.869 & $3.06 \pm .54$ & -1.580 \\
\hline & 3학년 & $3.67 \pm .55$ & $(.002)$ & $2.96 \pm .35$ & $(<.001)$ & $3.18 \pm .60$ & (.115) \\
\hline \multirow[t]{3}{*}{ 연령 } & 25세 미만 & $3.49 \pm .59$ & 2.541 & $2.83 \pm .35$ & 2.136 & $3.09 \pm .54$ & .210 \\
\hline & 25세이상-29세 이하 & $3.46 \pm .64$ & $(.081)$ & $2.90 \pm .32$ & $(.120)$ & $3.14 \pm .69$ & $(.811)$ \\
\hline & 30세 이상 & $3.83 \pm .50$ & & $2.99 \pm .29$ & & $3.03 \pm .56$ & \\
\hline \multirow[t]{5}{*}{ 종교 } & 불교 & $3.47 \pm .66$ & .382 & $2.86 \pm .33$ & 1.252 & $3.21 \pm .60$ & 1.413 \\
\hline & 기독교 & $3.45 \pm .67$ & (.821) & $2.92 \pm .39$ & (.289) & $3.09 \pm .55$ & (.230) \\
\hline & 카톨릭 & $3.58 \pm .67$ & & $2.89 \pm .37$ & & $3.10 \pm .50$ & \\
\hline & 없음 & $3.53 \pm .54$ & & $2.81 \pm .33$ & & $3.06 \pm .55$ & \\
\hline & 기타 & $3.68 \pm .26$ & & $2.84 \pm .31$ & & $2.77 \pm .56$ & \\
\hline \multirow{3}{*}{ 건강상태 } & 좋다 & $3.57 \pm .58$ & 7.433 & $2.89 \pm .34$ & 7,497 & $3.12 \pm .55$ & 1.343 \\
\hline & 보통이다 & $3.32 \pm .53$ & $(.001)$ & $2.70 \pm .33$ & $(.001)$ & $2.97 \pm .54$ & (.263) \\
\hline & 나쁘다 & $3.07 \pm .70$ & & $2.68 \pm .19$ & & $3.17 \pm .78$ & \\
\hline 대학생활 & 만족하다 & $3.65 \pm .57$ & 10.465 & $2.95 \pm .33$ & 16.580 & $3.16 \pm .56$ & 2.868 \\
\hline \multirow[t]{2}{*}{ 만족도 } & 보통이다 & $3.36 \pm .54$ & $(<.001)$ & $2.74 \pm .30$ & $(<.001)$ & $3.06 \pm .55$ & $(.059)$ \\
\hline & 불만족하다 & $3.25 \pm .71$ & & $2.70 \pm .36$ & & $2.89 \pm .60$ & \\
\hline \multirow[t]{3}{*}{ 학교성적 } & 상 & $3.82 \pm .61$ & 9.829 & $2.97 \pm .40$ & 3.107 & $3.15 \pm .73$ & .423 \\
\hline & 중 & $3.49 \pm .56$ & $(<.001)$ & $2.83 \pm .32$ & $(.046)$ & $3.10 \pm .52$ & (.656) \\
\hline & 하 & $3.27 \pm .61$ & & $2.81 \pm .37$ & & $3.03 \pm .58$ & \\
\hline 간호과 & 고교성적을 고려해서 & $3.11 \pm .32$ & 2.389 & $2.65 \pm .20$ & 5.076 & $2.91 \pm .46$ & 1.333 \\
\hline \multirow[t]{6}{*}{ 지원동기 } & 적성과 취미에 맞아서 & $3.69 \pm .59$ & $(.029)$ & $3.03 \pm .38$ & $(<.001)$ & $3.21 \pm .63$ & (.243) \\
\hline & 취업률이 높아서 & $3.51 \pm .59$ & & $2.82 \pm .30$ & & $3.05 \pm .55$ & \\
\hline & 부모님의 권유로 & $3.34 \pm .63$ & & $2.72 \pm .36$ & & $3.07 \pm .55$ & \\
\hline & 봉사직을 갖기 위해 & $3.42 \pm .48$ & & $2.84 \pm .21$ & & $2.96 \pm .49$ & \\
\hline & 해외로 갈 기회가 많아서 & $3.65 \pm .41$ & & $2.75 \pm .07$ & & $2.87 \pm .44$ & \\
\hline & $\begin{array}{l}\text { 본잉이 가족의 질화이나 입 } \\
\text { 원시에 받은 인상 때문에 }\end{array}$ & $3.47 \pm .47$ & & $2.87 \pm .31$ & & $3.34 \pm .36$ & \\
\hline 간호학 & 만족하다 & $3.63 \pm .55$ & 18.776 & $2.95 \pm .34$ & 32.331 & $3.17 \pm .57$ & 6.112 \\
\hline 전공에 & 보통이다 & $3.29 \pm .55$ & $(<.001)$ & $2.64 \pm .22$ & $(<.001)$ & $2.92 \pm .48$ & $(.003)$ \\
\hline 대한 만족도 & 불만족하다 & $2.76 \pm .79$ & & $2.52 \pm .31$ & & $3.01 \pm .78$ & \\
\hline 실습량에 & 만족하다 & $3.61 \pm .62$ & 7.546 & $2.96 \pm .36$ & 14.763 & $3.27 \pm .59$ & 12.390 \\
\hline \multirow[t]{2}{*}{ 대한 만족도 } & 보통이다 & $3.51 \pm .51$ & $(.001)$ & $2.78 \pm .28$ & $(<.001)$ & $3.00 \pm .40$ & $(<.001)$ \\
\hline & 불만족하다 & $3.23 \pm .62$ & & $2.70 \pm .32$ & & $2.86 \pm .67$ & \\
\hline 간호학생에 & 만족하다 & $3.53 \pm .59$ & .171 & $2.90 \pm .36$ & 4.319 & $3.20 \pm .58$ & 5.790 \\
\hline 대한 환자의 & 보통이다 & $3.48 \pm .59$ & (.843) & $2.77 \pm .32$ & $(.014)$ & $3.01 \pm .43$ & (.003) \\
\hline 인식과 태도 & 불만족하다 & $3.50 \pm .67$ & & $2.83 \pm .26$ & & $2.89 \pm .74$ & \\
\hline
\end{tabular}


4. 자기효능감, 전문직 자아개념 및 임상실습 만족도와의 상관관계

대상자의 자기효능감과 전문직 자아개념 $(\mathrm{r}=.595$, $\mathrm{p}<.001)$ 간에는 유의한 정적 상관관계가 있는 것으 로 나타났다. 그리고 대상자의 전문직 자아개념과 임상실습 만족도(r=.396, $\mathrm{p}<.001)$ 간에는 유의한 정 적 상관관계가 있는 것으로 나타났다<표 4>.

<표 4> 자기효능감, 전문직 자아개념 및 임상실습 만족도와의 상관관계

\begin{tabular}{ccc}
\hline & 자기효능감 & $\begin{array}{c}\text { 전문직 } \\
\text { 자아개몀 }\end{array}$ \\
\hline \hline 전문직 자아개념 & $\begin{array}{c}.595 \\
(p<.001)\end{array}$ \\
임상실습 만족도 & $\begin{array}{c}.082 \\
(p=.170)\end{array}$ & $\begin{array}{c}.396 \\
(p<.001)\end{array}$ \\
\hline
\end{tabular}

5. 임상실습 만족도에 영향을 미치는 요인

대상자의 임상실습 만족도의 주요 예측 요인을 파악하기 위하여 단계적 회귀분석을 실시하였다. 자기효능감은 임상실습 만족도와 유의한 상관관계 나타나지 않아 분석에 투입하지 않았다. 성별은 더 미변수 처리하여 분석에 이용하였다. 다중회귀 분
석결과, 회귀모형은 통계적으로 유의하게 나타났고 $(\mathrm{F}=14.230, \mathrm{p}<.001)$, 설명력은 $21 \%$ 였다. 간호대학생 의 임상실습 만족도에 대한 유의한 예측요인은 전 문직 자아개념 $(\mathrm{p}<.001)$, 실습량에 대한 만족도 $(\mathrm{p}=.017)$, 간호학생에 대한 환자의 인식과 태도 $(\mathrm{p}=.050)$ 로 나타났고, 이 중에서 전문직 자아개념 이 가장 큰 예측요인으로 확인되었다<표 5>.

\section{$\mathrm{N}$. 고찰 및 결론}

본 연구는 3 년제 간호과 학생 278 명을 대상으로 임상실습에서의 자기효능감, 전문직 자아개념과 임 상실습 만족도와의 관계를 파악하고 자기효능감과 전문직 자아개념이 임상실습 만족도에 미치는 영 향정도를 파악하고자 시도되었다. 연구대상자의 $69.0 \%$ 가 간호학전공에 대해 만족하였는데 이는 대 상자의 $46.0 \%$ 가 취업률이 높아서, $23.0 \%$ 가 적성과 취미에 맞아서와 같은 본인의 의지에 의해 간호과 를 지원했다는 데서 그 이유를 찾을 수 있다. 대상 자의 $11.2 \%$ 가 간호학생에 대한 환자의 인식과 태 도에 불만족하다고 보고하였는데 이는 실습학생과 환자와의 대인관계를 어렵게 하여 간호역할수행에 도 영향을 줄 것으로 생각된다.

<표 5> 임상실습 만족도에 영향을 미치는 요인에 대한 회귀분석

\begin{tabular}{|c|c|c|c|c|c|c|c|c|}
\hline \multirow{2}{*}{ 요인 } & \multicolumn{8}{|c|}{ 임상실습 만족도 } \\
\hline & B & SE & $\beta$ & $\mathrm{t}$ & $p$ & $\mathrm{R}^{2}$ & $\mathrm{~F}$ & $p$ \\
\hline 상수 & 67.224 & 13.171 & & 5.104 & .000 & & & \\
\hline 전문직 자아개념 & .632 & .116 & .336 & 5.460 & .000 & & & \\
\hline 성별(남자) & -5.768 & 3.302 & -.096 & -1.747 & .082 & & & \\
\hline 전공에 대한 만족도 & 1.176 & 2.021 & .036 & .582 & .561 & .207 & 14.230 & .000 \\
\hline 실습량에 대한 만족도 & -3.440 & 1.438 & -.146 & -2.392 & .017 & & & \\
\hline $\begin{array}{l}\text { 간호학생에 대한 환자의 } \\
\text { 인식과 태도 }\end{array}$ & -2.863 & 1.456 & -.112 & -1.966 & .050 & & & \\
\hline
\end{tabular}


실습학생의 $14.8 \%$ 와 $19.1 \%$ 가 환자 및 보호자와 의 대인관계가 어렵다고 보고한 강현옥[16]과 양진 주[17]의 연구도 이와 같은 맥락으로 볼 수 있다. 실습학생에 대한 환자의 인식과 태도를 변화시킬 수 있는 개선방안과 교육방안이 마련되어야 할 것 으로 생각된다. 본 연구에서는 학생의 $17.2 \%$ 가 실 습량에 불만족을 나타냈는데 이는 3년제와 4년제 학생을 대상으로 한 강현옥[16]의 연구에서의 $33.1 \%$ 와 3 년제 간호학생을 대상으로 한 서원희[8] 연구에서의 $54.1 \%$ 와 차이가 있었다. 총 임상실습 시간은 같지만 서원희[8]의 연구는 실습을 모두 마 친 학생을 대상으로 하였고 본 연구는 실습을 반 정도 끝낸 2학년이 $69.1 \%$ 를 차지하여 대상자가 주 관적으로 느끼는 실습량의 차이로 인해 나타난 결 과로 생각된다.

자기효능감의 평균은 3.50 점으로, 3 년제 간호과 학생들을 대상으로 한 김영희[13]의 연구에서의 3.70점보다는 낮게 나타났으나 4년제 간호학과 학 생들을 대상으로 한 황성자[4]의 연구에서의 3.52 점과는 비슷하게 나타났다. 자기효능감은 간호학생 에게 임상실습을 성공적으로 수행할 수 있다는 자 신감을 가지게 하고 적극적으로 참여하게 하는 동 기를 부여하므로[18] 교육과정에서 학생들의 자기 효능감을 증진시킬 수 있는 방안이 요구된다.

전문직 자아개념의 평균은 2.85 점으로 강현옥 [16]과 서원희[8]의 연구에서의 2.7점보다 높았으나 이정애[9]의 2.90 점보다는 낮았다. 이와 같은 결과 는 대상자의 선정과 표집과정의 차이와 더불어 대 상자의 특성 및 실습환경, 가치관 등의 여러 가지 요인들에 의해 영향을 받기 때문으로 생각된다. 하 위영역에서는 간호기술이 2.94점으로 가장 높게 리 더십이 2.79점으로 가장 낮게 나타나 선행연구 [8][16]에서의 결과와 차이가 있었다.

임상실습 만족도의 평균은 3.10점으로 서원희[8] 의 연구에서 2.60 점과 강현옥[16]의 연구에서 2.90 점보다 높아 본 연구대상자들이 실습에 만족하고 있는 것으로 평가되었다. 하위영역에서 실습지도
점수가 2.88점으로 가장 낮게 나타나 김영희[13]의 연구결과와는 대조적이었다. 실습지도 외의 모든 영역에서는 평균 3.00점 이상으로 나타나 실습 전 임교수 확충과 같은 실습지도 방식의 변화를 통해 실습만족도를 더 높일 수 있을 것으로 생각된다.

자기효능감은 성별, 학년, 건강상태, 대학생활의 만족도, 학교성적, 간호학 선택동기, 전공만족도, 실습량에 대한 만족도 및 간호학생에 대한 환자의 인식과 태도에서 차이가 있는 것으로 나타났다. 김 영희도 임상실습에서 남학생의 자기효능감이 유의 하게 높게 나타나 본 연구와 같은 결과를 보였으 나[13] 남학생수가 여학생수보다 훨씬 적은 경우이 므로 본 결과를 확신하는데 무리가 있다고 본다. 그러나 소수이기는 하지만 임상현장에서 겪게 되 는 다양한 경험들이 남자 간호학생에게 자신감을 주는 기회가 된 것으로 생각된다[13]. 학년에서도 3학년이 통계적으로 유의하게 높게 나타나 황성자 의 연구결과[4]와 일치하였는데, 이는 학년이 올라 가면서 간호에 대한 기술이나 지식에 자신감이 향 상되어 나타난 결과로 보여진다. 김영희는 간호학 선택동기와 전공만족도가 자기효능감과는 차이가 없다고 하여 본 연구와는 다른 결과를 제시하였고 [13], 황성자는 학년과 간호학 만족도가 자기효능 감에 있어 유의한 차이가 있다고 하여 본 연구결 과를 지지하고 있다[4].

전문직 자아개념은 성별과 학년에 따라 차이가 있었으며 건강상태, 대학생활의 만족도, 학교성적, 간호학 선택동기, 간호학 전공에 대한 만족도, 실 습량에 대한 만족도, 간호학생에 대한 환자의 인식 과 태도에서 차이가 있었다. 강현옥도 전문직 자아 개념이 성별, 대학생활 만족도, 학교성적, 간호학 선택동기, 임상실습 만족도, 전공에 대한 만족도, 실습량에 대한 만족도에서 유의한 차이가 있다고 하여[16] 본 연구결과를 지지하였다. 또한 이정애 는 전문직 자아개념이 전공에 대한 만족도에서만 차이를 보였으며[9] 하나선과 박효미는 간호학생의 전문직 자아개념은 전공만족도와 더불어 의사결정 
과 간호사에 대한 소명의식 및 대인관계와도 관련 이 있다고 하여[19] 전문직 자아개념은 많은 영향 요인이 있음을 재확인하였다. 또한 학생들의 전문 직 자아개념을 높이기 위해서는 간호교육자나 간 호전문가들이 올바른 전문직 자아개념을 가지고 학생들의 역할모델이 되어야한다고 하였다[19].

임상실습 만족도를 살펴보면 성별과 간호학 전 공에 대한 만족도, 실습량에 대한 만족도 및 간호 학생에 대한 환자의 인식과 태도에서 차이가 있는 것으로 나타났으나 한수정은 임상실습 만족도는 교육내용의 확실성에서 차이를 보이고 다른 특성 에서는 차이를 보이지 않았다고 하였다[6]. 김영희 는 일반적 특성과 임상실습 만족도와는 차이가 없 다고 하여[13] 본 연구결과와는 다른 견해들을 보 이고 있다. 이는 만족도는 주관적인 판단이므로 개 인마다 느끼는 것에 차이가 있을 수 있어 나타난 결과로 보여진다.

본 연구 변수들 간의 상관관계를 살펴보면 자기 효능감은 전문직 자아개념과 유의한 정적 상관관 계가 있었고 임상실습 만족도와는 유의한 상관관 계를 보이지 않았는데 김영희[13]와 신은주[18]의 연구에서는 자기효능감이 임상실습 만족도와 유의 한 정적 상관관계를 보인다고 하여 본 연구결과와 차이가 있어 추후연구가 필요하다고 본다. 전문직 자아개념은 임상실습 만족도와 유의한 정적 상관 관계를 나타나 김문실[20]과 서원희[8]강현옥[16]의 연구 결과와 일치하였다. 이정애[9]는 간호학생의 전문직 자아개념 발달에 임상실습 교육이 영향을 미치는 것으로 보고하고 있어 교육이나 상담 등의 프로그램을 통해 학생들의 전문직 자아개념을 증 진시키는 방안이 마련되어야 할 것이다.

회귀분석을 통해서 전문직 자아개념은 임상실습 만족도의 가장 영향력 있는 예측요인으로 나타났 다. 본 연구에서 자기효능감이 임상실습 만족도에 영향을 주는 요인으로 확인되지는 않았지만, 간호 학생은 임상실습에서 자기효능감이 높을수록 전문 직에 대한 자아개념이 높으며 전문직 자아개념이
높을수록 임상실습 만족도가 더 높아진다는 것이 확인되었다. 그러나 최근 몇년 동안 간호학과 학생 수의 증가는 학생들의 임상실습환경을 양적, 질적 으로 열악하게 만들고 있다. 이는 간호과 학생들의 자기효능감을 떨어뜨리고 전문직 자아개념에도 영 향을 줄 것이며 결국 임상실습만족도까지도 낮아 지게 만들 수 있다는 것을 의미한다. 이는 더 나아 가 간호학생들이 졸업 후 전문직업인으로서 간호 사의 역할을 수행하는 데에도 영향을 미칠 것으로 사료된다. 따라서 간호학생이 임상실습을 통해 이 와 같은 점들을 충족시킬 수 있는 임상실습 중재 방안이 조속히 마련되어야 할 것으로 생각된다. 간 호과 학생들에게 조직적이고 체계적인 임상실습을 적용함으로써 간호기술을 효율적으로 습득하여 간 호학생들의 임상실습에서의 만족도가 극대화될 것 이며 졸업 후 전문직 간호사로서의 만족도까지 향 상시키게 될 것이다.

본 연구를 통해 간호학생들의 전문직 자아개념 은 임상실습 만족도에 가장 큰 영향을 미치는 변 수임이 확인되었다. 전문직 자아개념은 자기효능감 과 유의한 정적 상관관계가 있으므로 자기효능감 이 높으면 전문직 자아개념이 높다. 따라서 간호학 생의 임상실습 만족도를 높이기 위해서는 전문직 자아개념을 높일 수 있는 구체적인 임상실습 중재 방안이 모색되어야 할 것이다. 본 연구결과를 토대 로 다음과 같은 제언을 하고자 한다.

간호학생의 전문직 자아개념을 증진시킬 수 있 는 프로그램의 개발이 필요하다.

간호학생의 자기효능감과 임상실습 만족도와의 관계를 검증하는 추후연구가 필요하다.

\section{참고문헌}

1. Chan, D.(2002), Development of the clinical learning environment inventory: using the 
theoretical framework of learning environment studies to assess nursing students' perceptions of the hospital as a learning environment, Journal of Nursing Education, Vol.41(2);69-75.

2. Nath, C., Schmidt, R., \& Gunel, E.(2006), Perceptions of professionalism vary most with educational rank and age, Journal of Dental Education, Vol.70(8);825-834.

3. 박미영, 김순이(2000), 전문대 간호학생들의 첫 임 상실습 체험 연구, 한국간호교육학회지, Vol.6(1); 23-35.

4. 황성자(2006), 간호대학생들의 임상실습 스트레스, 자기효능감 및 자아존중감과의 관계, 한국간호교 육학회지, Vol.12(2);205-213.

5. 이윤정, 김귀분(2008), 임상간호사의 이직경험, 대 한간호학회지, Vol.38(2);248-257.

6. 한수정(2000), 임상실습 시 간호학생이 지각하는 힘 북돋우기(empowerment)와 실습만족도에 관한 연구, 한국간호교육학회지, Vol.6(1);132-146.

7. Bandura, A.(1986), Social foundations of thought and action: A social cognitive theory, Englewood Cliffs, New Jersey: Prentice-Hall Inc, p.617.

8. 서원희(2006), 간호전문대학생의 간호전문직 자아 개념과 임상실습 만족도, 경북대학교 대학원 석사 학위 논문, pp.9-22.

9. 이정애(2004), 간호대학생의 전문직 자아개념과 간 호사의 대한 이미지, 간호행정학회지, $\mathrm{Vol} .10(3)$; 325-334.

10. Arthur, D.(1995), Measurement of the professional self-concept of nurses: developing a measurement instrument, Nurse Education Today, Vol.15(5);328-325.

11. 강윤숙, 조희, 노영숙, 부은희, 안금희(2006), 학교 -병원 협력실습교육 프로그램이 간호학생의 임상 실무수행능력과 만족도에 미치는 효과, 한국간호 교육학회지, Vol.12(1);13-20.

12. 성미혜(2010), 간호대학생의 학제 간 성취동기,
임상수행능력 및 임상실습만족도의 관련성 비교 연구, 기본간호학회지, Vol.17(1);90-98.

13. 김영희(2009), 일 대학 간호학생의 임상실습 교육 에 대한 자기효능감, 자기주도성 및 임상실습만족 도와의 관계, 기본간호학회지, $\mathrm{Vol} .16(3) ; 307-315$.

14. Sherer, M., Maddux, J.E., Mercandante, B., Prentice-Dunn, S., Jacobs, B., \& Rogers, R.W.(1982), The self- efficacy scale: construction \& validation. Psychological Reports, Vol.51(2); 663-671.

15. 홍혜영(1995), 완벽주의 성향, 자기효능감, 우울과 의 관계연구, 이화여자대학교 석사학위 논문, pp.27-28.

16. 강현옥(2009), 간호학생의 임상수행능력과 간호전 문직 자아개념 및 임상실습 만족도, 계명대학교 대학원 석사학위 논문, pp.26-30.

17. 양진주(2009), 간호학생의 임상수행능력에 영향을 미치는 요인, 한국간호교육학회지, Vol.15(2);159165.

18. 신은주(2008), 일 대학 간호학생의 자기효능감, 실습만족도 및 기본간호수기술과의 관계, 기본간 호학회지, Vol.15(3);380-386.

19. 하나선, 박효미(2009), 간호학생이 지각한 전문직 사회화가 역할갈등 및 전문직 자아개념에 미치는 영향, 임상간호연구, Vol.15(2);47-56.

20. 김문실, 임은주(2006), 서울소재 4년제 간호대학 생들의 자아개념과 임상실습 만족도, 이화간호학 회지, No.40;116-124.

접수일자 2011년 9월 10일 심사일자 2011년 9월 19일 게재확정일자 2011년 11월 12일 